\title{
The difference between a probability and a probability density
}

\author{
Antje Kohnle, ${ }^{1}$ Alexander Jackson, ${ }^{1}$ and Mark Paetkau ${ }^{2}$ \\ ${ }^{1}$ School of Physics and Astronomy, University of St Andrews, St Andrews, KY16 9SS, United Kingdom \\ ${ }^{2}$ Department of Physical Sciences, Thompson Rivers University, Kamloops, Canada V2C, OC8
}

Learning introductory quantum physics is challenging, 1 in part due to the different paradigms in classical mechanics and quantum physics. 2] Classical mechanics is deterministic in that the equations of motion and the initial conditions fully determine a particle's trajectory. Quantum physics is an inherently probabilistic theory in that only probabilities for measurement outcomes can be determined. Prior to studying quantum physics, students will typically have little experience with probabilistic analyses of physical systems, and thus probability may be a conceptual hurdle for introductory quantum physics students. 3, 4, This article describes two interactive simulations developed as part of the QuVis Quantum Mechanics Visualization Project 5-7 that aim to bridge the gap between classical mechanics and quantum physics using probabilistic analyses of classical systems. The simulations illustrate how a probability density can be obtained for two classical systems well known to students. The key learning goals of the simulations are to introduce students to probability densities and to help students distinguish between a probability and a probability density. The simulations build on previous work by Bao and Redish 3 . who developed an activity that used pseudo-random video frames of a glider in harmonic motion to derive a classical probability density for this system, and a University of Washington quantum mechanics tutorial focusing on probability and probability density for a classical system. 8] Interactive simulations allow students to easily carry out experiments and change variables that would be difficult to do with real equipment, and help students connect multiple representations by showing explicitly how they are linked. 9] The simulations described here only require basic knowledge of algebra and classical mechanics. They run on touchscreen devices as well as desktop computers, and can be run in a standard web browser from the $\mathrm{QuV}$ is website or downloaded for offline use.

Probability densities of continuous variables like position are of fundamental importance in quantum physics. For a particle confined to one dimension, the position probability density gives the probability per unit length of finding the particle as a function of position. The probability of finding the particle over an interval is the area under the probability density curve for the given interval. As the total probability of finding the particle anywhere must equal one, the probability density is normalized. Probabilities have no unit, must be numbers between zero and one, and the total probability must equal one. The position probability density in one dimension has unit $m^{-1}$ ("probability per unit length") and can in general have a numerical value that is greater than one. While the probability of finding the particle at a single point is zero, the probability density at a point will in general have a non-zero value. The two simulations described aim to help students distinguish between a probability and a position probability density using familiar classical systems.

\section{OVERVIEW OF THE SIMULATIONS}

Fig. 1 shows a screenshot of the Probabilistic analysis of a mass-spring system simulation. 10 The simulation shows a mass on a spring in harmonic motion and a photographer who takes snapshots of the block's position at random times. Once a photo is taken, the position of the block is determined. This increments the histogram below the mass-spring system, which shows the number of snapshots binned into position intervals (see Fig. 1). If a large number of snapshots are taken, one finds more snapshots close to the turning points where the block is moving more slowly, and fewer snapshots around the equilibrium position $x=0$ where the block is moving most quickly. The buttons in the lower right panel allow students to turn the snapshot number histogram into a probability density histogram. The observed probability density $P$ for each bin in this histogram is determined as the number of snapshots $N_{B i n}$ in that bin divided by the total number of snapshots $N_{T o t}$ and the length $\Delta x$ of the bin. Thus, the observed probability density

$$
P=\frac{N_{\text {Bin }}}{N_{\text {Tot }} \Delta x} \text {. }
$$

The number of snapshots in each bin and thus the observed probability density histogram is subject to statistical fluctuations which on average decrease with an increasing number of snapshots. Students can also display the theoretical probability density histogram in the limit that the number of snapshots tends to infinity, decrease the bin width, and display the theoretical probability density curve in the limit that the bin size tends to zero. The simulation includes short texts in the top right panel explaining the displayed quantities which can be shown by pressing the "?" buttons (see Fig. 1).

The "Challenges" tab in the simulation allows students to solve multiple challenges aligned with the learning goals. For example, students need to determine the probability of finding the block in a certain interval from a given snapshot number histogram or a given probability density histogram. Students thus need to determine the area under the probability density histogram over the 


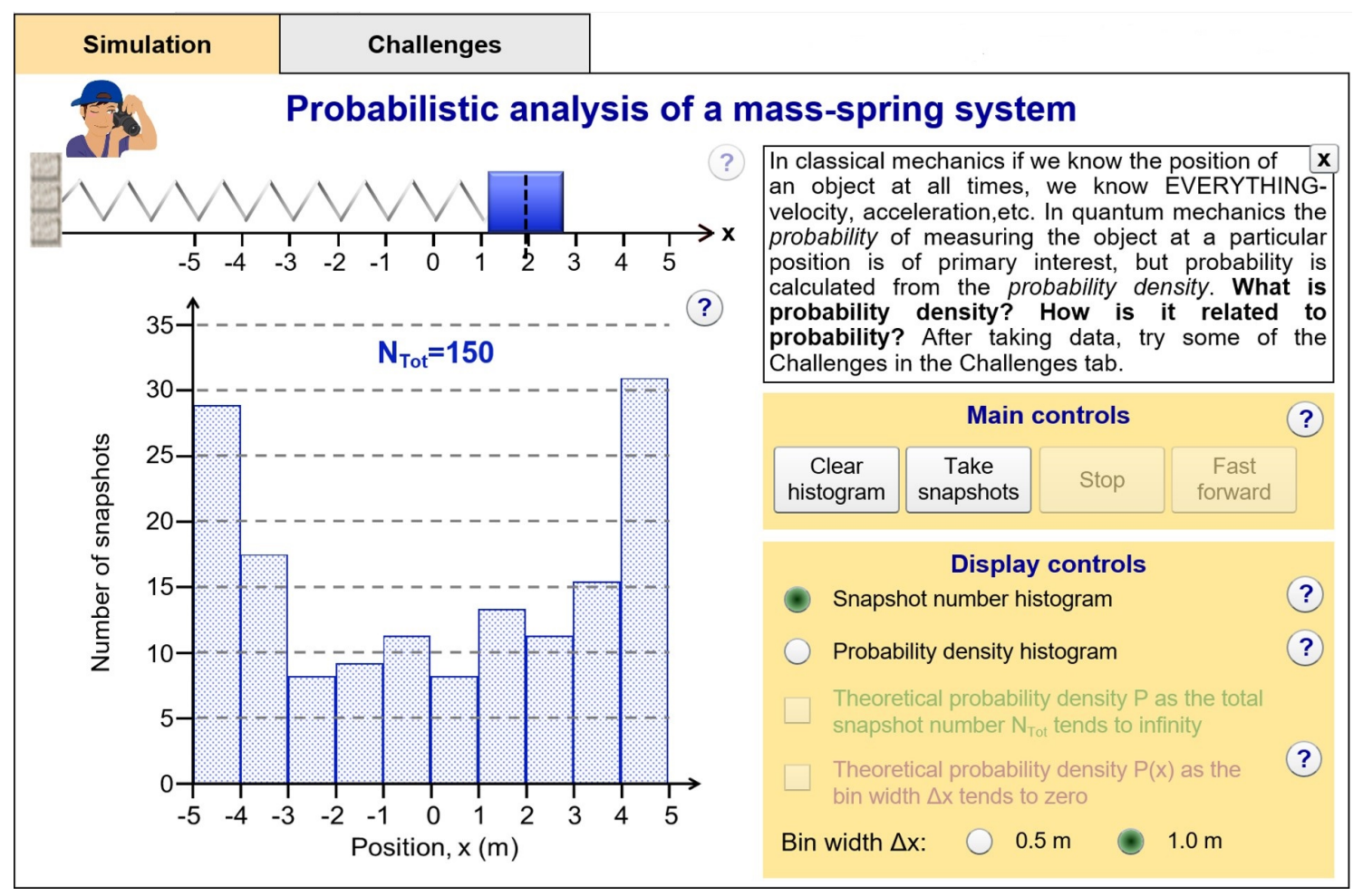

FIG. 1. A screenshot of the Probabilistic analysis of a mass-spring system simulation showing a snapshot number histogram obtained from taking photos of the block's position at random times.

given interval. Challenges can be solved in any order, include a score counter, and give feedback on submitted answers.

Fig. 2 shows part of the Probabilistic analysis of a block on a track simulation. [11] In this simulation, students can adjust the widths and heights of two horizontal regions of a frictionless track while a block with $30 J$ of mechanical energy moves back and forth along the track. A photographer takes photos of the block's position at random times. The resulting snapshot number histogram can be converted into a probability density histogram. Students can also overlay the theoretical probability density curve, shown in Fig. 2 as the green and purple lines in the middle panel. As the block's speed is constant in each of the two regions, the theoretical probability density has the same value at all points in a given region.

Since the mechanical energy is constant, the higher the track in a given region, the slower the speed of the block and thus the greater the probability density in this region. The theoretical probability density for each region can be calculated as the probability of finding the block in this region divided by the length of the region. The theoretical probability Prob $_{1}$ of finding the block in the left region of the track is given by

$$
\operatorname{Prob}_{1}=\frac{t_{1}}{T / 2},
$$

where $t_{1}=L_{1} / v_{1}$ is the time needed for the block to traverse the left region, $L_{1}$ is the length of this region, $v_{1}$ is the speed of the block in this region and $T / 2=$ $L_{1} / v_{1}+L_{2} / v_{2}$ is the time needed for a full traversal of the track, which equals half the period $T$. Thus, the theoretical probability density in the left region is given by

$$
P_{1}=\frac{\operatorname{Prob}_{1}}{L_{1}}=\frac{2 t_{1}}{T L_{1}}=\frac{2}{T v_{1}},
$$

and is inversely proportional to the block's speed in this region. The speeds of the block in the different regions are given in the simulation.

The top panel in Fig. 2 shows the theoretical probability density for each of the two regions as well as the probability of the block being found in each of the two regions. The probability of finding the block in each region is given by the product of the probability density value and the length of the region. As shown in Fig. 2 , it is thus possible that even though the probability density value is smaller in the left region, the probability of finding the block is greater in the left region due to its greater length.

The "Challenges" tab in the simulation allows students to solve multiple challenges, e.g. to set up a track for which the probability of finding the block is as small as possible in the left region, or for a given track to determine the theoretical probability density in a region from the block's speeds in the two regions and the heights of 


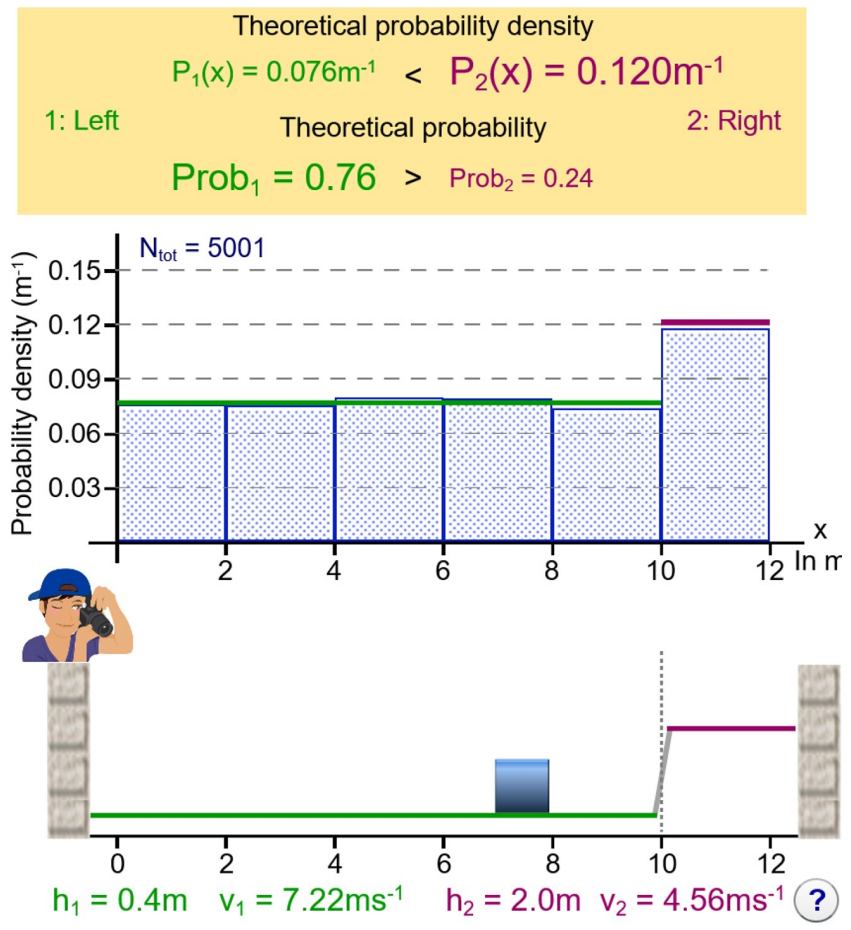

FIG. 2. Part of a screenshot of the Probabilistic analysis of a block on a track simulation, showing the track (bottom), the experimentally determined and theoretical probability density histogram (middle), as well as the theoretical probability and probability density values for both sides of the track (top).

the regions.

\section{IMPLEMENTATION}

We have used the simulations shown in Figs. 1 and 2 since 2014 and 2016 respectively in introductory and intermediate-level quantum courses at the University of St Andrews, with several hundred students in total $(N=350)$. The simulations are embedded into activities (see Appendix for download instructions) which cover a variety of tasks ranging from qualitative descriptions to collecting data and simple calculations. 12]

The activity for the Probabilistic analysis of a massspring system simulation includes the following tasks:

- Determine how the speed of the oscillator is qualitatively related to the number of snapshots in a bin.

- Show how the snapshot number histogram is translated into a probability density histogram using an example from your collected data.

- Explain the difference between the observed and the theoretical probability density histogram.

- Describe the limiting procedure to find the theoretical probability density curve.
Preliminary evidence supporting the usefulness of these simulations for learning has also been obtained. The fraction of students correctly answering each of the Probabilistic analysis of a mass-spring system activity questions for $2014(N=67), 2015(N=60)$ and 2016 $(N=70)$ was determined. The majority of responses (70\%-90\% depending on question) were fully correct. Course assessment in 2016 following the Probabilistic analysis of a block on a track simulation included a question with a similar setup to the simulation and the following statements: "If the probability in one region is larger than in another region then the probability density in the first region must also be larger.", and "The probability densities for the two regions have to add up to one, just like the probabilities, because the block has to be somewhere.". More than $85 \%$ of students $(N=88)$ disagreed with these statements and were able to explain why each of the statements is incorrect (the size of the region relates the probability density and the probability, and it is the area under the probability density curve which must be one respectively).

We also use these simulations to compare and contrast them with the case of a one-dimensional quantum particle in a box, a system commonly discussed in introductory quantum physics courses. The classical probability densities in these simulations arise due to a lack of knowledge of the initial conditions. We assume that we do not know the position of the block, but can make probabilistic statements about the block's position based on its speed in different regions. This is a case of classical ignorance: while the block has a definite position at each point in time, we do not know this position. In contrast, in the standard Copenhagen interpretation of quantum physics, the quantum particle in a box is not moving back and forth in the box; it does not have a definite position at each point in time. Thus, the probability density of the quantum particle describes the quantum uncertainty of the particle's position, which does not have a definite value prior to measurement.

In conclusion, the two interactive simulations shown in Figs. 1 and 2 with accompanying activities are useful in bridging classical and quantum physics through a probabilistic analysis of classical systems familiar to students. The simulations introduce students to probability densities and help students distinguish between probabilities and probability densities. The QuVis website [7] includes interactive simulations on further topics suitable for introductory quantum physics, e.g. on the infinite square well, multiple particles in an infinite square well, and interferometer experiments with single photons.

\section{APPENDIX}

Simulations, activities, and solutions can be downloaded from the $\mathrm{QuVis}$ website. [7. Simulations can be run from the website or downloaded using the download symbol appearing below the simulation thumbnail. The 
pdf icons below the simulation thumbnails link to the activities, while editable solutions (password protected) are accessed via the lock symbols. Instructors interested in obtaining the password for the activity solutions or helping with evaluation studies are requested to email quvismail@st-andrews.ac.uk.

\section{ACKNOWLEDGMENTS}

The authors would like to thank Sam Lloyd for the coding of the Probabilistic analysis of a block on a track simulation, and all students that participated in the evaluation studies and provided feedback on the materials. We thank the University of St Andrews for funding the development of simulations.
[1] K. Krijtenburg-Lewerissa, H. J. Pol, A. Brinkman, and W. R. van Joolingen, "Insights into teaching quantum mechanics in secondary and lower undergraduate education," Phys. Rev. Phys. Educ. Res. 13(1), 010109 (2017).

[2] E. Marshman and C. Singh, "Framework for understanding the patterns of student difficulties in quantum mechanics," Phys. Rev. ST Phys. Educ. Res. 11(2), 020119 (2015).

[3] L. Bao and E. F. Redish, "Understanding probabilistic interpretations of physical systems: A prerequisite to learning quantum physics," Am. J. Phys. 70, 210-217 (March 2002).

[4] D. Domert, C. Linder and A. Ingerman, "Probability as a conceptual hurdle to understanding one-dimensional quantum scattering and tunnelling," Eur. J. Phys. 26, 47-59 (January 2004).

[5] A. Kohnle, I. Bozhinova, D. Browne, M. Everitt, A. Fomins, P. Kok, G. Kulaitis, M. Prokopas, D. Raine and E. Swinbank, "A new introductory quantum mechanics curriculum," Eur. J. Phys. 35, 015001 (January 2014).

[6] A. Kohnle, C. Baily, A. Campbell, N. Korolkova and M. J. Paetkau, "Enhancing student learning of two-level quantum systems with interactive simulations," Am. J.
Phys. 83, 560-566 (June 2015).

[7] The Quantum Mechanics Visualization Project. Www. st-andrews.ac.uk/physics/quvis

[8] Tutorials in Physics: Quantum Mechanics. depts. washington.edu/uwpeg/tutorials-QM

[9] C. E. Wieman, W. K. Adams, P. Loeblein and K. K. Perkins, "Teaching Physics Using PhET Simulations," Phys. Teach. 48, 225-227 (April 2010)

[10] Probabilistic analysis of a mass-spring system. $\quad$ ww.st-andrews.ac.uk/physics/quvis/ simulations_html5/sims/ClassicalOscillator/ ClassicalOscillator.html

[11] Probabilistic analysis of a block on a track. www.st-andrews.ac.uk/physics/quvis/simulations_ html5/sims/block-on-track/block-on-track.html

[12] Probabilistic analysis of a mass-spring system activity. www.st-andrews.ac.uk/physics/quvis/simulations_ html5/problems/Problems_h10_Classical0scillator. pdf Probabilistic analysis of a block on a track activity. www.st-andrews.ac.uk/physics/quvis/simulations_ htm15/problems/Problems_h40_block-on-track.pdf 